\title{
Stamp Forming of Polypropylene based Polymer-Metal Laminates: The Effect of Process Parameters on Spring Back
}

\author{
Wentian Wang ${ }^{1, a}$, Sudharshan Venkatesan ${ }^{1, b}$, Anthony Sexton ${ }^{1, c}$ and Shankar \\ Kalyanasundaram ${ }^{1, d}$ \\ ${ }^{1}$ Research School of Engineering, Australian National University, Canberra, ACT, 0200, Australia \\ awentian.wang@anu.edu.au, bsudharshan.venkatesan@anu.edu.au canthony.sexton@anu.edu.au \\ 'shankar.kalyanasundaram@anu.edu.au
}

Keywords: Spring Back, Polymer Metal Laminate (PML), Stamp Forming.

\begin{abstract}
This paper investigates the effect of process parameters such as Blank Holder Force (BHF) and Feed Rate, on the spring back behavior of a polymer metal laminate (PML) system comprised of aluminum and polypropylene. Specimens were formed over a hemispherical punch in stamp forming process. A novel real time strain measuring system, ARAMIS, was employed to capture the strain evolution during forming. The results of this work indicate that both BHF and feed-rate exert influence in PML spring back behavior. Fundamental correlation between strain evolution during spring back and the shape of the finished part will be presented. A major finding from this work is that aluminum dominates the spring back behavior of PML in stamp forming.
\end{abstract}

\section{Introduction}

The PML structure consists of two metal sheet outer layers and a polymer sheet center core. The advantage of PML is that it saves weight while maintaining the equivalent stiffness ${ }^{[1]}$, which makes it a viable alternative to steel. Today, automobile parts like doors, fenders and interior panels have been manufactured from laminate sheets by the automotive industry ${ }^{[2]}$. Also, due to their superior mechanical properties, laminate sheets have been applied in a number of aerospace and marine applications ${ }^{[3]}$. Spring back phenomenon is one of the major sources leading to geometry inaccuracy in material forming and therefore, a better understanding on this phenomenon is desired in the present and past last decades for design and manufacturing purposes ${ }^{[4]}$. This paper investigates the spring back behavior of PML in stamp forming process by correlating strain evolution during unloading to the final shape of the formed part. The two process paramters that includes the BHF and Feed Rate are varied to study the effect of spring back. These two parameters have been proven to have important influence in stamp forming of laminate material systems ${ }^{[5-8]}$. Many studies have been conducted to reduce the spring back in forming through numerical analysis as well as experiments. Factors such as mechanical properties of the material, tool curvature radii, layer-thickness ratio and contact friction have been proven to be able to influence material spring back in forming ${ }^{[9-11]}$. The open die design of the press machine facilitates the application of ARAMIS to capture strain evolution during forming and this is world first for this class of material systems.

\section{Experimental Procedure}

Materials and PML Preparation. The polymer metal laminate structure analysed in the project consisted of two $0.6 \mathrm{~mm}$ thick $5005 \mathrm{H} 34$ aluminium outer layer sheets and $1 \mathrm{~mm}$ thick plain polypropylene sheet in between. Two layers of Glucofilm ${ }^{\mathrm{TM}} 5000$, a $50 \mu \mathrm{m}$ thick hot-melt polypropylene adhesive layer, were used to bond the polymer metal laminate. The bonding and melting temperature of Glucofilm is $150^{\circ} \mathrm{C}$ and $160^{\circ} \mathrm{C}$, respectively. Before gluing with polypropylene, $5005 \mathrm{H} 34$ aluminium sheets were etched by immersing into the $5 \% \mathrm{NaOH}$ solution for 5 minutes followed by rinsing in clean water to eliminate the material impurities as well as surface dirt. 
During the gluing process, two pieces of aluminum sheets, two pieces of adhesives and one piece of polypropylene were stacked together in a sandwich structure before been placed in the heat press machine. Heat press machine heated up samples to $155^{\circ} \mathrm{C}$ which reaches bonding temperature of the adhesives while less than the melting point of polypropylene. Machine was set up to press one sample at a time with $300 \mathrm{kPa}$ pressure, maintained for 2 minutes. The water coming from the water tap connecting to the heat press machine then cooled the samples down to $80{ }^{\circ} \mathrm{C}$ at a rate of $50-70^{\circ} \mathrm{C} / \mathrm{min}$ before removal. In the cooling process, $300 \mathrm{kPa}$ pressure was maintained to ensure the laminate structure was adhered properly. All manufactured samples were then cut into a circular shape with a diameter of $180 \mathrm{~mm}$ by water-jet cutting which was controlled by computer to achieve precision in cutting.

Experimental Setup. The press machine consists of a 30-ton H-frame, $100 \mathrm{~mm}$ diameter hemispherical punch and an open die with a diameter of $105 \mathrm{~mm}$ was employed to conduct stamp forming experiments of PML. The process control and data logging computer controls the feed rate as well as punch displacement through hydraulic feed controller, the dynamics of which was provided by a 20 liter accumulator, charged using a two stage pump locating next to the machine. A $150 \mathrm{kN}$ compression load cell recorded punch force and a $250 \mathrm{~mm}$ linear potentiometer measured displacement during forming. During the experiments, force and displacement were logged at rates of $20 \mathrm{~Hz}$ and $100 \mathrm{~Hz}$, respectively.

The open die design of the press allows the strain measurement using ARAMIS three dimensional strain measuring system developed by GOM mbH, Germany. The application of ARAMIS system in stamp forming experiments provides considerable information on the surface deformation during experiments. Two CCD cameras are used to capture the surface deformation using three dimensional photogrammetry methodologies. In order to obtain the ideal condition for ARAMIS system measurement, a stochastic pattern with a white background and blank speckles on top is applied to the sample surface. This strain measuring system is very accurate with error of less than $0.02 \%$ in strain measurements.

Experimental Design. All samples were formed to a depth of $12 \mathrm{~mm}$ to ensure that most samples could be formed. During the experiments, the ARAMIS system was set up to capture 6 frames every second. This frame rate is able to capture enough images in the short forming period. This work analyzes the spring back effect on PML through two different forming conditions, namely, blank holder force and feed rate. For each of these process parameters, three different level values were selected and are illustrated in table 1. A full factorial Design of Experiments (DOE) methodology was adopted in conducting the experiments.

Table 1: Process Conditions and Levels

\begin{tabular}{c|c}
\hline \hline Forming Condition & Level \\
\hline Blank Holder Force $(\mathrm{kN})$ & $2,7,14$ \\
Feed Rate $(\mathrm{mm} / \mathrm{s})$ & $20,40,60$ \\
\hline
\end{tabular}

Evaluation of Spring Back. Spring back is represented as a ratio of the change in vertical displacement during unloading to the vertical displacement at stable stage as shown in the following expression.

$$
\text { Spring back }=\frac{D_{\max }-D_{f}}{D_{\max }}
$$

where $D_{\max }$ is the maximum displacement of the PML at the end of stamp forming. $D_{f}$ is the final displacement after unloading. 


\section{Results and Discussion}

Effects of Process Parameters on Spring Back. Fig. 1. illustrates the major effect of process parameters carried out through DOE on the spring back of PML samples at pole. It is observed that large spring back was exhibited at low BHF and it drops when increasing BHF. High BHF leads to a reduced flowing of the blank into the die and hence the blank experiences larger mechanical strain. This in turn leads to a smaller ratio of recovered elastic strain to the plastic strain and is clearly demonstrated in Fig. 1.

In metal forming, the Feed Rate plays an insignificant role as metal is not a rate dependent material at room temperature. Due to the existence of polypropylene in PML samples, the Feed Rate can be a significant process variable due to the flow nature of the thermoplastic polypropylene [12]. However, it is essential to note that the Feed Rate has insignificant influence on the PML spring back behavior compared to that of the BHF. This leads to an important conclusion that the aluminum layer dominates PML in its spring back behavior.

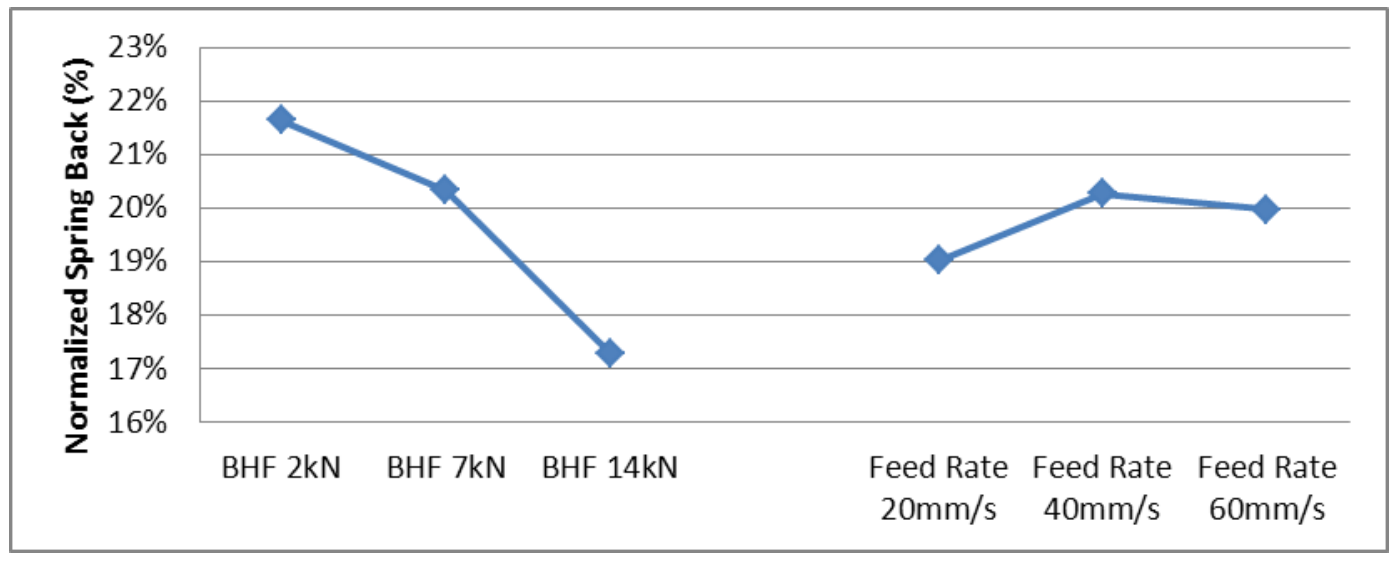

Fig. 1. Major effect of spring back behvior at pole

Strain Variation in Spring Back. Fig. 2.shows the strain value across the meridian line of the blank at the end of forming and after elastic recovery stage. There is a double peak shape on the strain along the meridian line through the centre of the testing sample and the maximum strain is caused by friction between the punch and blank [13].

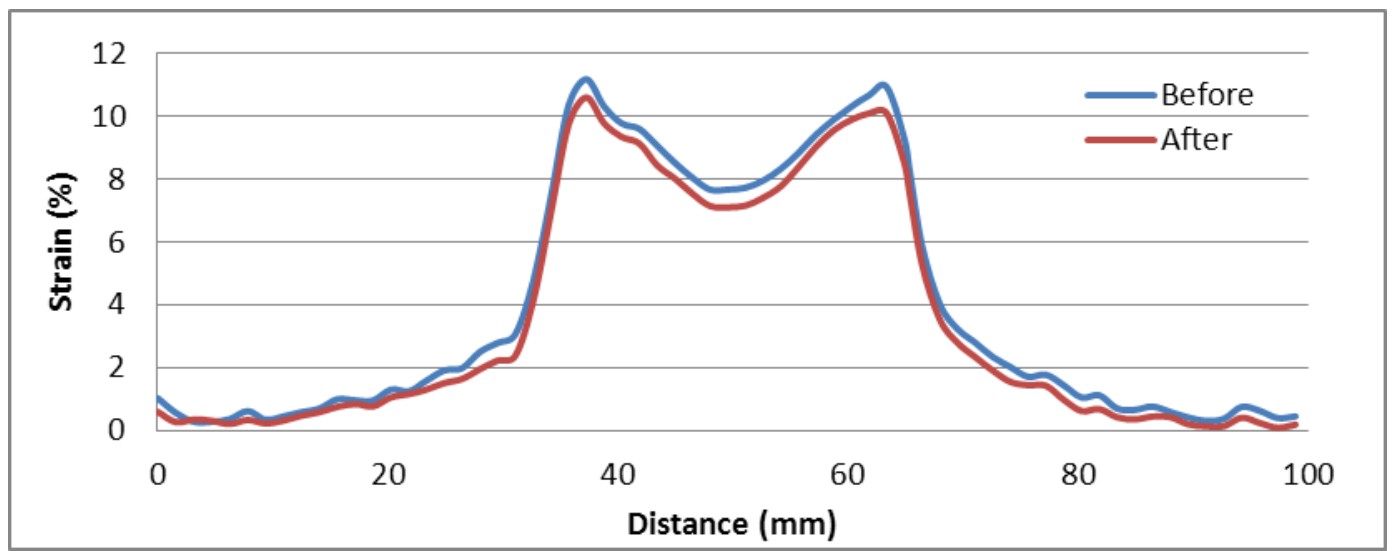

Fig. 2.Strain variation on meridian line of PML under 2kN BHF, 20mm/s Feed Rate

By comparing the spring back to the ratio of maximum elastic strain to the total strain across the blank, Fig. 3. demonstrates that there is a strong correlation between the percentage change in displacement and the ratio of maximum elastic strain to the total strain during spring back. This finding is one of the major contribution of this work where a fundamental correlation between the ratio of maximum elastic strain to the total strain has been used to correlate the shape errors due to spring back of the finished part. 


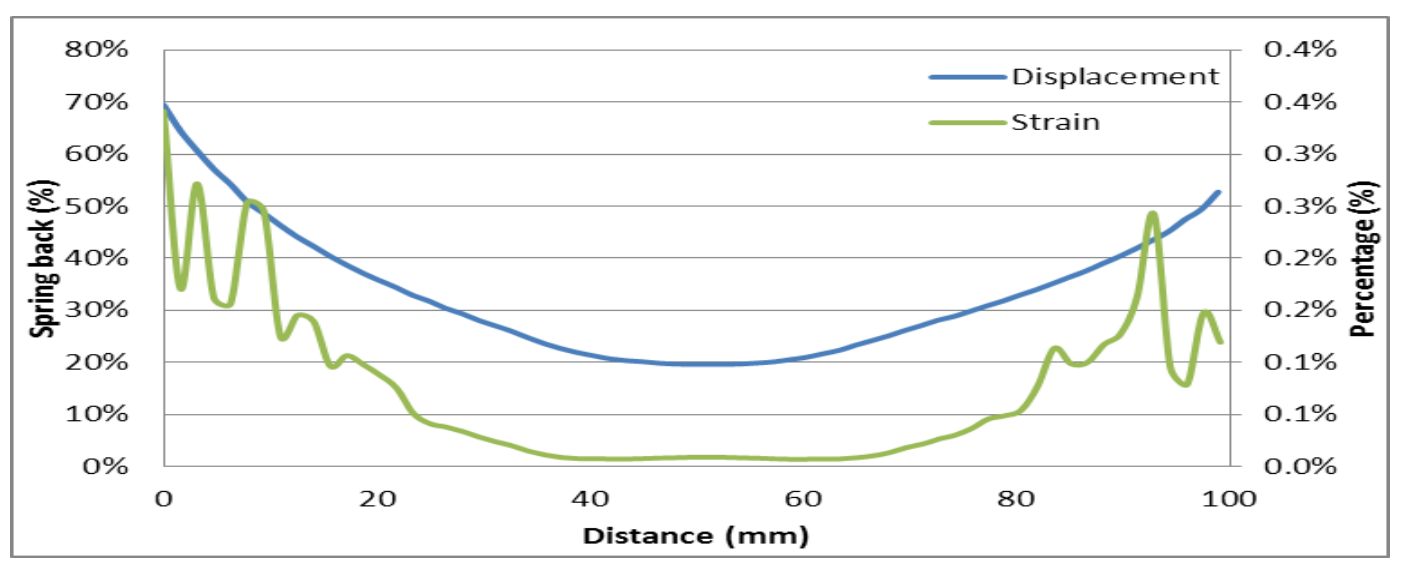

Fig. 3. Comparison between spring back and the percentage of elastic strain to the total strain

Spring Back Comparison between PML and its Constituent Material. To validate the hypothesis that the aluminium plays a dominating role in PML spring back behaviour, a spring back comparison was conducted between PML and its constituent material, 0.6mm thickness H34 5005 aluminium and $1 \mathrm{~mm}$ thickness plain polypropylene. All experiments were conducted at $2 \mathrm{kN}$ BHF and $20 \mathrm{~mm} / \mathrm{s}$ Feed Rate for consistency. According to Fig. 4, PML behaves much more similar to the aluminium than polypropylene, which helps to validate the hypothesis. A major conclusion that can be derived from this work is that the spring back behaviour of PML systems is dominated by its monolithic metal sheet when the ratio of stiffness between the metal and polymer layer is high.

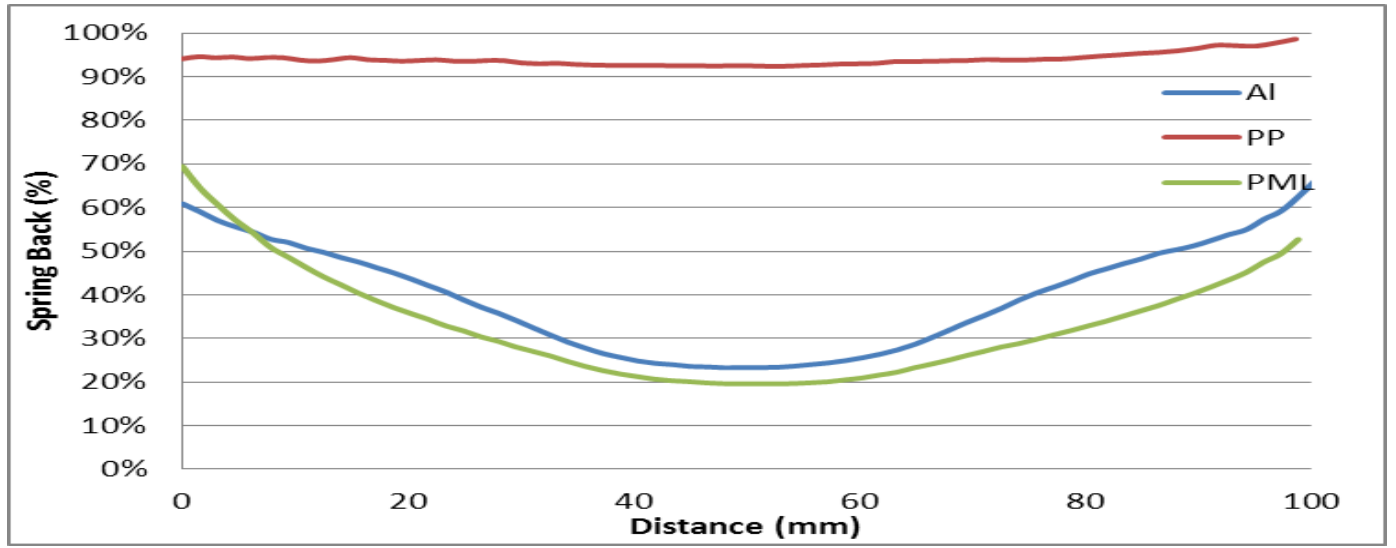

Fig. 4. Spring back comparison between PML and its constituent material

\section{Conclusion}

ARAMIS 3D strain measuring system was used to capture and compute the deformation process during the experiments as a part of the research. All experiments utilise an open die design press machine and use two three-dimensional cameras with high resolution to capture the evolution of specimen during forming. This research has shown that PML experiences less spring back at high $\mathrm{BHF}$ at pole since high BHF generates more plastic deformation on the specimen, which matches with the literature. Compare to BHF, Feed rate plays an insignificant role in PML spring back behaviour. Furthermore, there is a severe wrinkling appearing at flange region at $2 \mathrm{kN}$ and this phenomenon is diminished and cannot be detected by visual inspection at high BHFs. It is worth noting that experimental results prove the existence of the correlation between spring back and material elastic recovery. By comparing the spring back behaviour of PML and that of its constituent material, it is concluded that monolithic aluminium layer dominates the spring back behaviour of PML. This finding suggests that PML systems would behave similarly to its monolithic metal layer in spring back due to the superior stiffness properties of metal layer compared to the polymer layer.

These results are essential experimental bases which can be applied for validation of finite element analysis of spring back on PML in stamp forming. The findings of this research enhance the 
understanding of stamp forming of PML material system especially in spring back, which facilitates the application of this class of material systems in the automotive industry and to other mass production applications.

\section{References}

[1] Lutz et al. 1986, High heat metal polymer laminate, US Patent 4,601,941.

[2] CK Chao, CH, Liu, HJ Wu. 1993. "A study of the stretching process of steel-polymersteel laminate. ” Journal of Materials Processing Technology. 155-172.

[3] R. Kopp, M. Nutzmann, J. Van Santen. 2005. Formability of lightweight, vibration damping and medium perfused sandwich sheets, analysis through practical experiments and FEM simulations. In: Thomsen OT et al., editors. Sandwich structures 7: advancing with sandwich structures and materials. Springer; 2005. p. 723-32.

[4] CA. Queener, RJ. De Anglelis. 1957 "Elastic spring back and residual stresses in sheet metal formed by bending." Trans ASME;61:757-68.

[5] L. Mosse W. Cantwell M.J. Cardew-Hall P. Compston and S. Kalyanasundaram 2006 "The influence of process variables on the formability of fibre-metal laminates", Journal of Material Processing Technology, vol. 172, pp. 163-168.

[6] A. Sexton W. Cantwell and S. Kalyanasundaram 2012 "Stretch forming studies on fibre metal laminate based on a self-reinforcing polypropylene composite", Composite Structures, vol. 94, pp. 431-437.

[7] L. Mosse W. Cantwell M.J. Cardew-Hall P. Compston and S. Kalyanasundaram 2005 "Effect of process temperature on the formability of fibre-metal laminates", Composites Part A: Applied Science and Manufacturing, vol. 36, no. 8, pp. 1158-66.

[8] J. Gresham W. Cantwell M.J. Cardew-Hall P. Compston and S. Kalyanasundaram 2006 "Drawing behaviour of Metal-Composite sandwich structures", Composite Structures, vol. 75, no. 1-4, pp. 305-312.

[9] R. Hino, Y. Goto, F. Yoshida. 2003 'Springback of sheet metal laminates in draw-bending.' The journal of Materials Processing Technology 341-347

[10] M.H Parsa et al. 2010 'Experimental and finite element study on the spring back of double curved aluminium/polypropylene/aluminium sandwich sheet.' Materials and Design $4174-4183$

[11] J.R Cho et al. 2003. 'Finite element investigation on spring-back characteristics in sheet metal U-bending process.' The Journal of Materials Processing Technology. 109-116.

[12] L. Mosse. 2005, 'Stamp forming of polypropylene based fibre-metal laminates: The effect of process variables on formability.' The Journal of Materials Processing Technology. 163-168.

[13] C. Morrow, S. Dharmalingam, S. Venkatesan, S. Kalyanasundaram, Stretch forming studies on thermoplastic composite, In: Proceedings of 6th Australiasian congress on applied mechanics, Perth, Australia December, 2010. 